Pramāṇa - J. Phys., Vol. 35, No. 6, December 1990, pp. L593-L598. (C) Printed in India.

\title{
A spiral growth model for quasicrystals in two dimensions
}

\author{
S BARANIDHARAN \\ Department of Physics, Indian Institute of Science, Bangalore 560012, India \\ MS received 17 July 1990; revised 25 September 1990
}

Abstract. Spiral space filling geometrical constructions using rhombuses in two dimensions are considered as plausible mechanisms for quasicrystal growth. These models will show staircase-like features which may be observed experimentally.

Keywords. Quasicrystals; tilings; five-fold symmetry.

PACS Nos 61.50; 61.55

\section{Introduction}

Quasicrystals with five-fold rotational symmetry discovered by Shechtman et al (1984) are still posing difficulties regarding an understanding of the inherent atomic ordering. Various models for their structure have been proposed and are being subjected to rigorous tests by diffraction techniques. At present the facet formations and growth mechanisms of quasicrystals are centres of interest. This is because different types of microstructures and morphologies have been observed in different alloys and sometimes in the same alloy. The pentagonal dodecahedral morphology was observed in $\mathrm{Ga}-\mathrm{Mg}-\mathrm{Zn}$ icosahedral phase (Ohashi and Spaepen 1987). The triacontahedral morphology was seen in $\mathrm{Al}-\mathrm{Li}-\mathrm{Cu}$ alloy (Dubost et al 1986; Kortan et al 1989a). For a variety of morphologies observed in the icosahedral phase like a flower, a rosette and coral, a torus, dendrite etc. reference is made to Janot and Dubois (1988). The morphology of a decagonal prism was found in $\mathrm{Al}-\mathrm{Cu}-\mathrm{Co}$ decagonal quasicrystal (Kortan et al 1989b). None of the various models proposed for the quasicrystals has been able to fully explain both the facet formation and growth of quasicrystals. The icosahedral glass model (Stephens and Goldman 1986) proposes that since icosahedral ordering is favoured in these Al-alloys (Bergman et al 1957), rapid solidification can freeze the liquid structure leading to a glass like formation with orientational order. Such a freezing from liquid state will result in an abnormal decrease in density (10\%), when compared to its crystalline form, owing to the void space between the icosahedral ciusters. In this model a growth mechanism for quasicrystal is not predicted and the quasicrystal is expected to be entropically stabilized. The Penrose tiling model (Levine and Steinhardt 1984; Kramer and Neri 1984; Jaric 1986; Socolar and Steinhardt 1986; Sasisekharan 1986; Elser 1987; Steinhardt and Ostlund 1987) proposes that there are two types of rhombohedra which arrange themselves quasiperiodically in three dimensions through a matching rule similar to the Penrose matching rule (Penrose 1974; Mackay 1982) for two dimensional tilings. This tiling model is supported by the observed triacontahedral morphology (Dubost et al 1986; Kortan et al 1989a). 
While this model predicts that the quasicrystal can be energetically stabilized (Ingersent and Steinhardt 1989), the contents of the rhombohedra are not known and the origin of their formation has not been explained. The twinning model (Carr 1985; Field and Fraser 1985; Khan and Wibbeke 1988; Vecchio and Williams 1988; Anantharaman 1989; Pauling 1989) is reported to be inconsistent with experiments (Cahn et al 1986) although growth twins may form during spiral growth in some cases.

In this work we see that stacking of geometrical shapes like rhombuses in a spiral fashion can lead to peculiar features which are relevant to an atomistic growth of quasicrystals and which can be experimentally tested at the microscopic level.

\section{Spiral growth of rhombuses with five-fold symmetry}

Some of the simple spirals in two dimensions are given by,

1. reciprocal $(r=a / \theta)$,

2. parabolic $(r=a \sqrt{\theta})$,

3. Archimedian $(r=a \theta)$,

4. Golden $\left(r=a \theta^{\tau}\right)$,

5. Logarithmic $\left(r=a e^{b \theta}\right)$ and

6. Exponential $(r=a \log (b \theta)$.

The generating functions are given in polar form where $a, b$ are constants. Bursill et al (1987) have schematically shown the simple spirals and also introduced the concept of spiral lattices. A spiral lattice is a discrete set of points distributed on a continuous spiral according to a quantization rule. A spiral structure is thus a discrete set of unit cells decorating a spiral lattice. A successive stacking of the unit cells on the spiral lattice represents the growth of the spiral structure.

We use the Archimedian spiral to generate the spiral lattice. The quantization rule, given as $\theta=2 \pi n / \alpha$ ( $\alpha$ is a constant), locates spiral lattice points at discrete values of $\theta$. The Archimedian spiral has been chosen in order to preserve linear variation in the spacings between various layers. There is no simple rule to associate a decoration at each spiral lattice point since there is an addition of intermediate lattice points with every layer (in other words the interval between adjacent $\theta$ values decreases with increasing layers). This problem can be circumvented by finding the distance between successive spiral lattice points and then deciding on the number of unit cells.

For a quasicrystal, growth is represented by a stacking of the thick and the thin rhombuses (whose vertex angles are 72 and 36 degrees respectively) in a spiral arrangement. A few seeds around which growth can take place are shown in figure 1. Other seeds similar to those in figure 1 behave in a similar manner during the spiral growth. In figure 1 the sprouts are also shown. We see that there is more than one possible sprout for each seed. Just as in the growth of regular crystals the sprouts are held to the seed by surface tension. In figure 2, a systematic spiral growth of one particular seed is shown in detail. We see that starting from a seed with five-fold symmetry there are ten branches (or spiral arms) each of which grows over its neighbouring branch. In this stacking process we have tried to preserve the direction of growth clockwise at each step. In other words, the growth is 'left-handed'. In figure 2 one can see local regions where rhombuses are arranged in periodic segments. These 'staircase' like structures which become larger in size in large tilings lead to an 

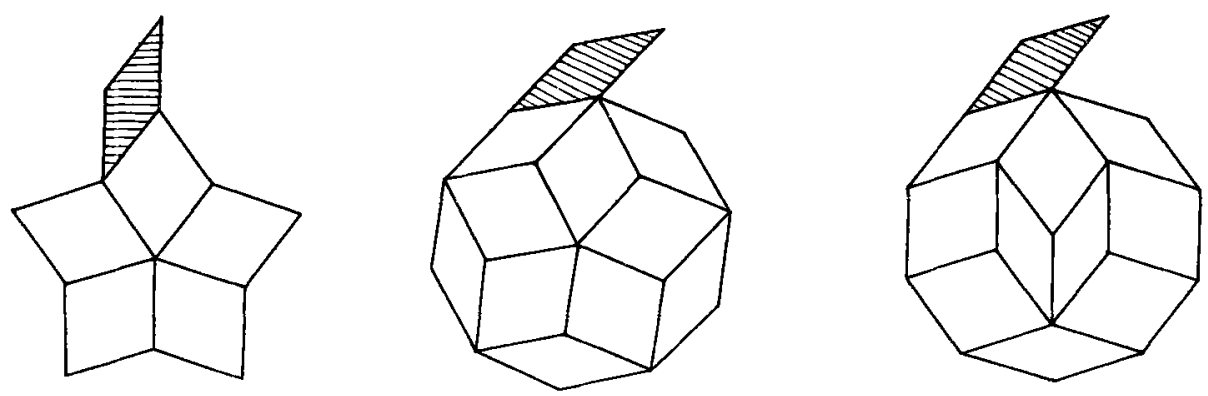

Figure 1. Some examples of nuclei (seeds) around which spiral growth can take place. The sprouts are shaded.
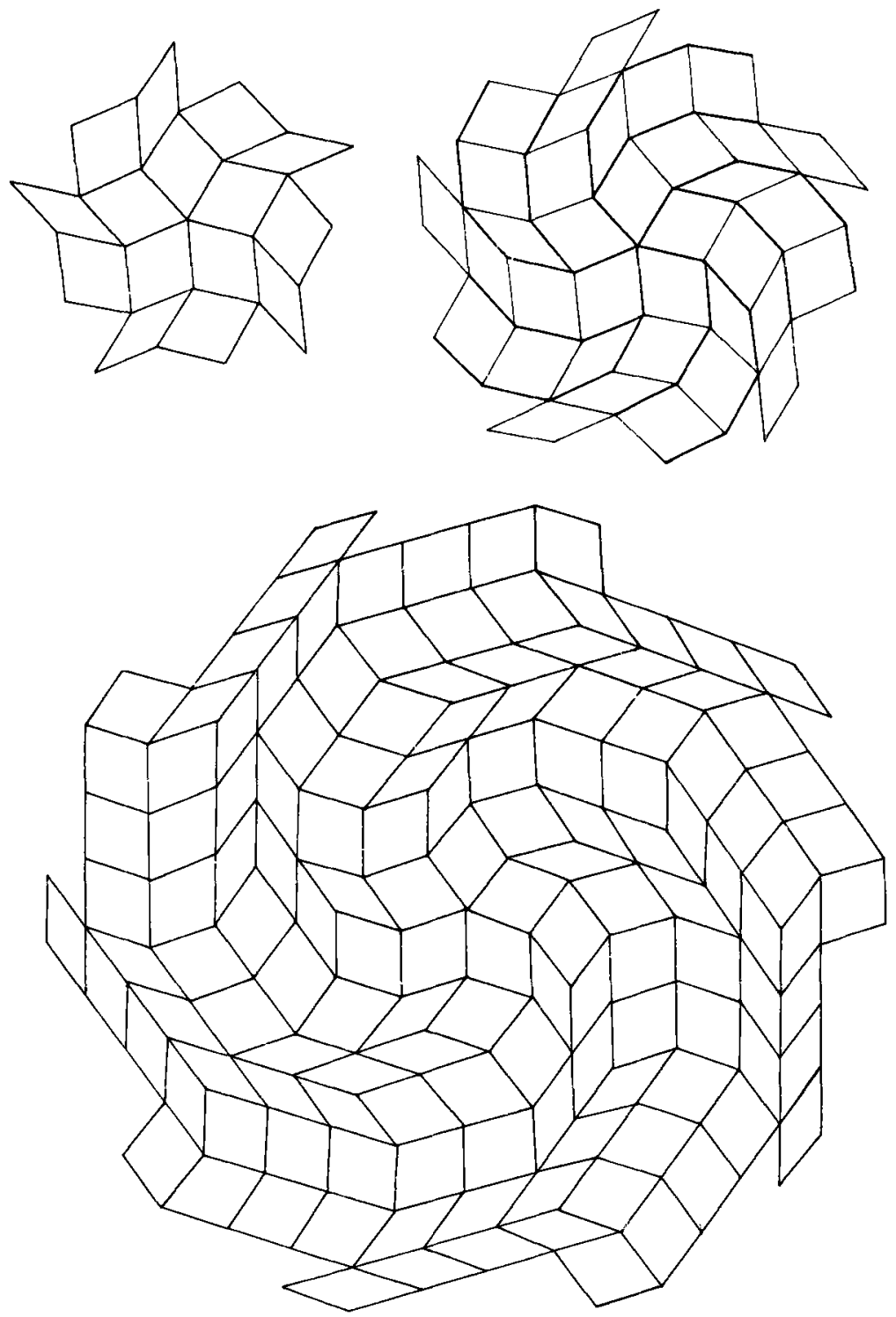

Figure 2. A star with five-fold symmetry is the nucleus. The ten armed growth results in staircases and an overall decagonal morphology. 


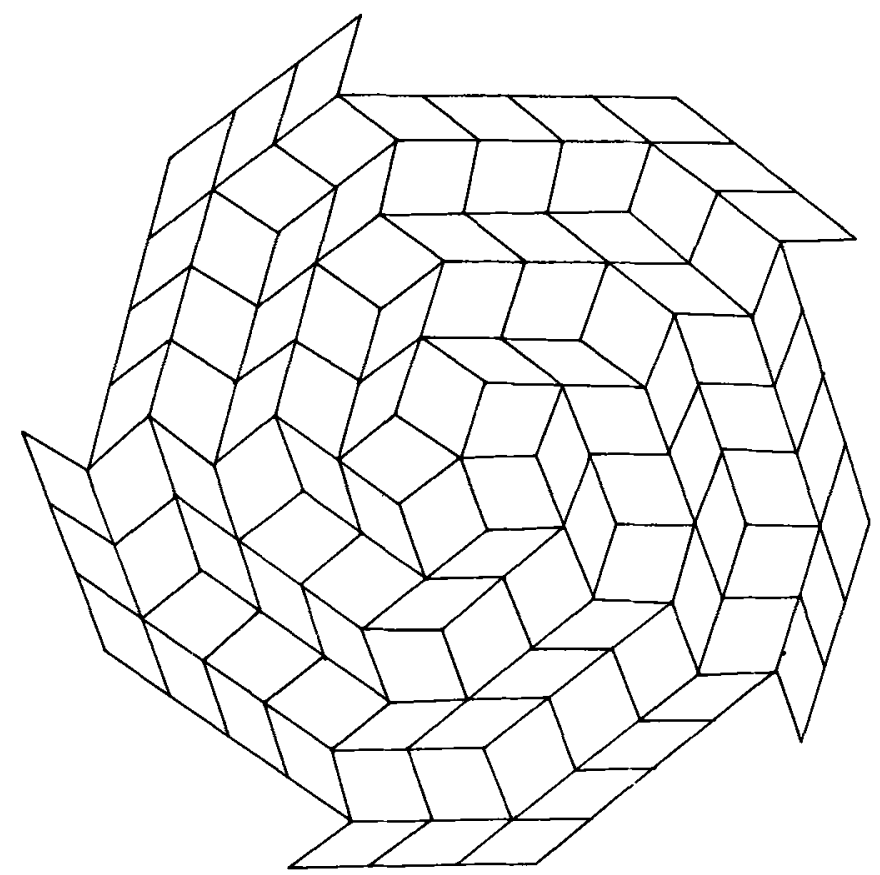

Figure 3. A closed decagon with five-fold symmetry is the seed. The growth is five branched and the morphology is a decagon.

overall decagonal morphology. The staircases can also be considered as finite sets of periodic leaflets in a growing branch.

Spiral growth has been attempted on different seeds. Figure 3 illustrates the growth of a seed which is a closed decagon with five-fold symmetry. While there are five growing branches, the morphology of the structure is a decagon. There are staircases as in figure 2 . In figure 3 there are five sets of vertices along five directions starting from the seed which are arranged periodically. A single spiral arm growth can be obtained if one uses the third seed as shown in figure 1 .

A careful inspection of figure 3 will reveal that there are larger and larger decagons around the centre whose edge lengths are in the ratio $1: 2: 3: \ldots$ etc. Thus this spiral growth can alternatively be thought of as a periodic inflation of the seed (which means the inflation operator will have rational roots) while the growth itself cannot be considered to be periodic. Hence we find that a rational or an integer root of an inflation operator does not guarantee a strict periodicity in the structure. We have earlier shown aperiodic tilings constructed by inflation operators whose roots are irrational (Sasisekharan et al 1989). When the seed is a star (like in figure 2) then there will be vertices forming larger and larger pentagons.

\section{Conclusions}

Spiral growth in conventional crystallography has been a well known model for crystal growth. Spiral tilings have also been considered in other contexts (Grunbaum 
and Shepherd 1988). Here one should mention a few other aspects of two dimensional spiral stacking of rhombuses which could be relevant to quasicrystal growth. Spiral growth will imply a local minimization of surface free energy. From the theory of tiling models one would believe that the staircase-like local ordering (parallel and adjacent rhombuses) will contribute to the configurational entropy of the quasicrystal. A numerical simulation study assuming two different types of atoms may answer the question whether the contribution from this entropy of mixing is significant. Spiral tilings will result in well defined morphologies and staircase-like features. Such staircases have indeed been observed recently by Chen et al (1990). They made these observations while studying an unannealed $\mathrm{Al}-\mathrm{Cu}-\mathrm{Co}$ decagonal phase using high resolution electron microscopy and disagreed with the simple Penrose tiling model for quasicrystals at the microscopic level. In three dimensions a spiral or helical growth of rhombohedra can be implanted into the existing models for quasicrystals. The formation of quasi-icosahedral spiral shapes in carbon particles during growth has been recently found in micrographs by Kroto and Mckay (1988) and their models in three dimensions possessed both icosahedral and helical features.

\section{Acknowledgements}

The encouragement and guidance of Prof E S R Gopal is gratefully acknowledged. This work is an outcome of a study based on the original remark by Prof V Sasisekharan on spiral structures in Physics and Biology. The discussions with Prof S Ranganathan have been fruitful. This letter is a part of a work on aperiodic tilings and quasicrystals funded by the Department of Science and Technology, Government of India.

\section{References}

Anantharaman T R 1989 Curr. Sci. 581067

Bergman G, Waugh J L T and Pauling L 1957 Acta Crystallogr. 10254

Bursill L A, Lin J P and Xudong F 1987 Mod. Phys. Lett. $5 \& 6195$

Cahn J W, Gratias D and Shechtman D 1986 Nature (London) 319102

Carr M J 1985 J. Appl. Phys. 591063

Chen H, Burkov S E, He Y, Poon S J and Shiflet G J 1990 Phys. Rev. Lett. 6572

Dubost B, Lang J M, Tanaka M, Sainfort P and Audier M 1986 Nature (London) 32448

Elser V 1987 Acta Crystallogr. A42 36

Field R D and Fraser H L 1985 Mater. Sci. Eng. 68 L17

Grunbaum B and Shepherd G C 1988 Tilings and Patterns (New York: Freeman)

Janot Ch and Dubois J M 1988 Quasicrystalline materials (Singapore: World Scientific)

Jaric M V 1986 Phys. Rev. B34 4685

Ingersent K and Steinhardt P J 1989 Phys. Rev. B39 980

Khan Y and Wibbeke H 1988 J. Mater. Sci. Lett. 7314

Kortan A R, Chen H S, Parsey J M Jr and Kimerling L C 1989a J. Mater. Sci. 241999

Kortan A R, Thiel F A, Chen H S, Tsai A P, Inoue A and Masumoto T 1989b Phvs. Rev. B40 9397

Kramer P and Neri R 1984 Acta Crystallogr. A40 580

Kroto H W and Mckay K 1988 Nature (London) 331328

Levine D and Steinhardt P J 1984 Phys. Rev. Lett. 532477

Mackay A L 1982 Physica A114 609

Ohashi W and Spaepen F 1987 Nature (London) 330555 
Pauling L 1989 Proc. Natl. Acad. Sci. 858595

Penrose R 1974 Bull. Inst. Math. Appl. 10266

Sasisekharan V 1986 Pramana - J. Phys. 26283

Sasisekharan V, Baranidharan S, Balagurusamy V S K, Srinivasan A and Gopal E S R 1989 Pramana - J. Phys. 31405

Shechtman D, Blech I, Gratias D and Cahn J W 1984 Phys. Rev. Lett. 531951

Socolar J E S and Steinhardt P J 1986 Phys. Rev. B34 617

Steinhardt P and Ostlund S 1987 The Physics of quasicrystals (Singapore: World Scientific)

Stephens P W and Goldman A I 1986 Phys. Rev. Lett. 561168

Vecchio K S and Williams D B 1988 Philos. Mag. B57 535 